\title{
Evaluación de antioxidantes en el té de hojas de camote morado (Ipomoea batatas L.)
}

\section{Antioxidant evaluation in the sweet potato purple (Ipomoea batatas L.) Leafs tea}

Rebeca Cervantes-Sierra ${ }^{1}$, Melquiades Barragán-Condori², Guadalupe Chaquilla-Quilca ${ }^{3}$

Fecha de recepción: 11 de diciembre de 2018

Fecha de aceptación: 14 de marzo de 2019

Cervantes-Sierra, R; Barragán-Condori, M; Chaquilla-Quilca,

G. Evaluación de antioxidantes en el té de hojas de camote morado (Ipomoea batatas L.). Tecnología en Marcha. Vol. 32-

4. Octubre-Diciembre 2019. Pág 51-59.

doi) https://doi.org/10.18845/tm.v32i4.4790

1 Estudiante, Escuela Académico Profesional de Ingeniería Agroindustrial, Facultad de Ingeniería, Universidad Nacional Micaela Bastidas de Apurímac, Perú.

(iD) https://orcid.org/0000-0003-1770-1586

2 Doctor en Ciencias. Departamento de Ciencias Básicas, Facultad de Ingeniería, Universidad Nacional Micaela Bastidas de Apurímac, Perú. (iD) https://orcid.org/0000-0001-6666-1301

3 Autor de correspondencia. Doctora en Ciencias. Escuela Académico Profesional de Ingeniería Agroindustrial, Facultad de Ingeniería, Universidad Nacional Micaela Bastidas de Apurímac, Perú. Correo electrónico: gchaquilla@unamba.edu.pe. (i) https://orcid.org/0000-0002-4994-9888 


\title{
Palabras clave
}

Hojas de camote morado; antioxidantes; tés herbales; subproductos agroindustriales.

\section{Resumen}

Las hojas de camote morado (Ipomoea batatas L.) un subproducto de la agroindustria, son utilizadas principalmente en la alimentación animal, en algunos países asiáticos son consumidas como vegetales en ensaladas; una nueva alternativa de uso para estas hojas en nuestra región podría ser mediante bebidas tipo té, a fin de extraer compuestos bioactivos presentes en sus hojas. Por lo que el objetivo del presente trabajo fue determinar los compuestos fenólicos y la capacidad antioxidante del té de hojas de camote morado (TCM), para ello se realizó una extracción acuosa a temperatura de ebullición de las hojas secas de camote morado proveniente del valle de Pachachaca, Abancay Apurímac-Perú, posteriormente se procedió a analizar, tomando como referencia al té verde comercial (TVC). Se determinó el contenido de Polifenoles totales por el método de Folin Ciucalteau, obteniéndose valores para TCM y TVC de 14.16 y $9.40 \mathrm{mg}$ de GAE/g de hojas respectivamente. También se evalúo la actividad antioxidante por 2 métodos químicos; el primero mediante la Capacidad antioxidante equivalente a Trolox (TEAC) con el ácido 2,2'-azino-bis-3-etilbenzotiazolina-6-sulfónico (ABTS) obteniéndose 8.61 y 7.98 umol Trolox/mL en TCM y TVC respectivamente, demostrando estos resultados que el TCM presenta mayor poder bioactivo y capacidad antioxidante; así mismo se evalúo la TEAC con 1,1-diphenyl-2-picrylhydrazyl (DPPH) reportando valores 25.16 y $25.63 \mu \mathrm{mol}$ Trolox/g para TCM y TVC, implicando estos resultados que el TCM presenta una capacidad antioxidante semejante al del TVC considerado por excelencia como bebida antioxidante. Se encontró además una correlación $r=0.9129$ entre polifenoles y antioxidantes.

\begin{abstract}
A purple sweet potato (Ipomoea batatas L.) leaves, a byproduct of agroindustry, mainly are used in animal feed, and also are consumed in salads as vegetable in Asian countries, a new use for these leaves in our region will be tea dinks, in order to extract bioactive compounds present in their leaves. The aim was to determine the phenolic compounds and antioxidant capacity the purple sweet potato leaves tea (TCM), an aqueous extraction at boiling temperature the dried leaves of purple sweet potato from Pachachaca valley Abancay-Apurímac, Perú was carried and analyzed, whit commercial green tea as a reference (TVC). The content of total polyphenols by Folin Ciucalteau was determined, obtaining 14.16 and $9.40 \mathrm{mg}$ of GAE/ g leaves for TCM and TVC respectively. The antioxidant activity by two methods was evaluated, the first one through antioxidant capacity equivalent to Trolox (TEAC) with 2,2'-azino-bis-3-etilbenzotiazolina6-sulfónico (ABTS), obtaining 8.61 and $7.98 \mu \mathrm{mol}$ Trolox/mL in TCM and TVC respectively, these result evidence that TCM exhibited better bioactive compounds and antioxidant capacity, likewise TEAC by 1,1-diphenyl-2-picrylhydrazyl (DPPH) was evaluated reporting 25.16 and $25.63 \mu \mathrm{mol}$ Trolox/g for TCM an TVC, implying that TCM display an antioxidant capacity similar to TVC considered an antioxidant beverage by excellence. A correlation $r=0.9129$ between polyphenols and antioxidant was also found.
\end{abstract}

\section{Keywords}

Purple sweet potato leaves; antioxidant; herbal teas; agroindustrial by-products. 


\section{Introducción}

Las hojas de camote (Ipomoea batatas L.) un subproducto de la agroindustria, ha sido utilizada como planta medicinal para enfermedades de la piel, también las hojas verdes son consumidas como vegetales en ensaladas en China [1], pero principalmente son utilizadas en la alimentación animal especialmente de cerdos ya sean frescas, secas o en ensilados debido a que presentan un considerable contenido de proteínas que van desde $25.5 \%$ BS. [2], hasta $37 \mathrm{mg} / 100 \mathrm{~g}$ BS, además que es una fuente de Vit $C, \beta$-carotenos, Hierro, Calcio, polifenoles y oxalatos, ya sea que se consuman frescas, secas o cocidas [3], también son ricas en carbohidratos, fibra dietaría, antioxidantes y otros micronutrientes deficientes en alimentos basados en el almidón, y que podrían balancear la dieta [4][5]. Diferentes estudios revelan que estas hojas son una fuente potencial de compuestos bioactivos [6] ya que poseen polifenoles y actividad antioxidante, como el reporte de un estudio en el que evaluaron extractos metanolicos de estas hojas, los mismos que contenían valores de fenoles totales de entre 2.78 a $5.35 \mathrm{~g}$ GAE/100 g BS [7], en otro estudio realizado en hojas de seis variedades de camote se obtuvieron 4.47 a $8.11 \mathrm{~g} \mathrm{GAE} / 100 \mathrm{~g}$ BS [8] también [1] reportaron $4.48 \mathrm{mg}$ GAE/g BS en extractos con diferentes solventes; así mismo también [9] evaluó la actividad antioxidante en extractos etanolicos con DPPH reportando valores de 38.6 $\mu$ mol Trolox/gr BH.

Varias investigaciones evidencian el incremento de polifenoles y la capacidad antioxidante de plantas cuando estos son sometidos a una extracción acuosa a temperatura de ebullición en bebidas tipo té, o infusiones caso de cedrón [10] té verde [11], infusiones de hierbas de Brasil como té negro, té verde, mate, menta, manzanilla anís entre otros [12] y extractos de 34 cafés comerciales puros y mezclados en Panamá [13].

Una alternativa en nuestra región, para diversificar el uso de estas hojas, podría ser mediante bebidas tipo té, a fin de extraer los compuestos bioactivos potenciales presentes; por lo que el objetivo de éste trabajo fue determinar compuestos fenólicos y capacidad antioxidante del té de hojas de camote morado en comparación con el té verde comercial.

\section{Metodología}

\section{Muestras y reactivos}

Las hojas de camote morado (Ipomoea batatas L.) provinieron del valle de Pachachaca Abancay Apurímac-Perú, una vez recolectadas fueron lavadas y secadas en una estufa a $65^{\circ} \mathrm{C}$ x $5 \mathrm{~h}$ y posteriormente almacenadas hasta su análisis (figuras 1 y 2),

Las hojas de té verde (Thea sinensis L.) de una marca comercial, fueron adquiridas en la ciudad del Cusco-Perú (figura 2).

Todos los reactivos fueron comprados de Merck (Darmastad, Alemania) sede Perú.

\section{Preparacion del extracto}

A ambas muestras se le realizó una extracción acuosa, con agua destilada a una relación 1:10, la cual se llevó a ebullición durante un minuto, se filtró, se aforó, se enfrió y se procedió a analizar a estos extractos sus componentes bioactivos.

Análisis de polifenoles totales (PFT)

La determinacion de polifenoles totales en los extractos fue determinada por espectrofotometría, basado en la reacción colorimétrica de óxido-reducción del reactivo Folin-Ciocalteu, empleando ácido gálico para la preparación de la curva estándar y utilizando un espectrofotómetro UV/VIS Genesys 10S (Thermo Scientific) descrito por [14] . 


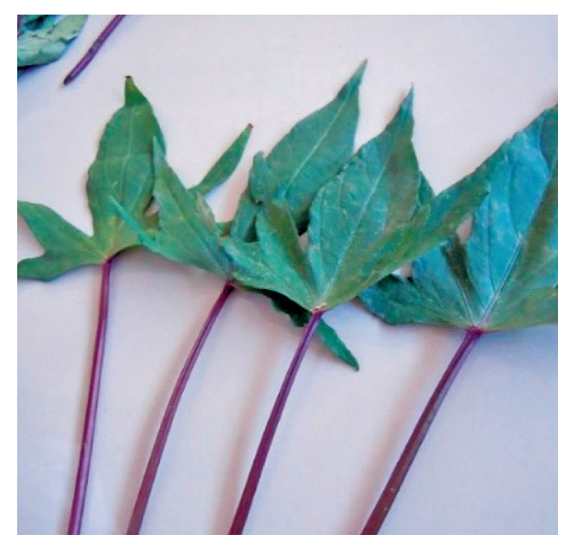

Figura 1. Hojas de camote morado (Ipomea batatas L.) frescas

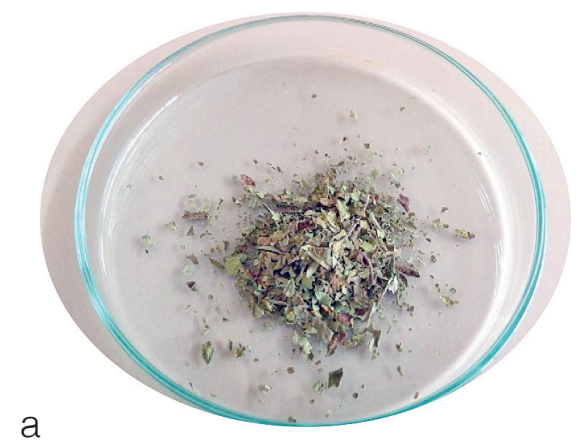

b

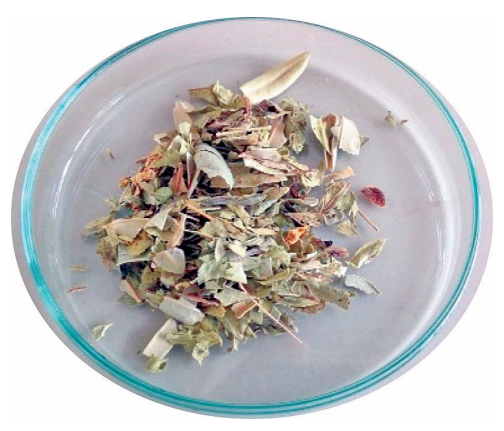

Figura 2. a) Hojas de camote morado (Ipomea batatas L.) secas; b) hojas de té verde (Thea sinensis L.) comercial secas.

\section{Determinacion de capacidad antioxidante TEAC ABTS}

Esta determinación está basada en la reducción del radical ABTS* (ácido 2,2'-azinobis-3-etilbenzotiazolina-6-sulfónico) por extractos que contienen compuestos antioxidantes utilizando como patrón el Trolox (ácido ( \pm )-6-hidroxi-2,5,7,8-tetrametil-croman-2- carboxilico), de acuerdo a [15] con algunas modificaciones. Inicialmente, el radical ABTS es generado haciendo reaccionar una solución de ABTS $7 \mathrm{mM}$ en agua con Persulfato de potasio $2.45 \mathrm{mM}$ en oscuridad durante 12-16 h y cuya absorbancia de este reactante se ajustó $0.700 \pm 0.02$ a temperatura ambiente a una longitud de onda de $734 \mathrm{~nm}$ en un espectrofotómetro UVNIS Genesys 10S (Thermo Scientific). Luego se agregan los extractos adecuadamente diluidos a la Solución ABTS* de modo que cuando se añaden $50 \mu \mathrm{L}$ del extracto a $3 \mathrm{~mL}$ de solución ABTS*, daría una inhibición del 20-80\% de absorbancia leída a 734 nm después de 10 minutos de agregada la muestra. La curva de calibración de Trolox se construyó midiendo la reducción en la absorbancia de la solución ABTS* a diferentes concentraciones de Trolox $(0-2000 \mu \mathrm{M})$. La actividad antioxidante se midió comparando con la curva patrón de Trolox, cuyos resultados se expresan como equivalentes Trolox. TEAC ABTS ( $\mu \mathrm{mol}$ Trolox $/ \mathrm{mL}$ ). Todas las determinaciones fueron realizadas por triplicado [16].

\section{Determinacion de Capacidad antioxidante TEAC DPPH}

La determinación de la capacidad antioxidante, se realizó utilizando como patrón de referencia el Trolox, en donde los extractos y el estándar actuaron reduciendo el radical 2,2-difenil-1picrilhidrazilo (DPPH). A $2.9 \mathrm{~mL}$ de solución de DPPH previamente ajustada a una absorbancia de 0.700 , se le adicionó $100 \mu \mathrm{L}$ del extracto acuoso o estándar, luego se agitó, se dejó incubar 
por 10 minutos a temperatura ambiente en oscuridad, luego se leyó en un espectrofotómetro UV/VIS Genesys 10S (Thermo Scientific) a $515 \mathrm{~nm}$ de longitud de onda. La calibración fue hecha con una solución de Trolox y la actividad antioxidante total fue expresada como micromoles de equivalentes Trolox por gramo de té. Utilizando etanol como blanco. Con respecto al radical DPPH se ha preparado a $50 \mu \mathrm{M}$ en $100 \mathrm{~mL}$ y de esta solución se ajusta a una absorbancia de $0.9( \pm 0.02)$ con etanol [17][18].

Análisis estadístico

Los resultados se procesaron mediante un análisis de varianza y las medias fueron comparadas con la prueba de Tukey con una significancia de 0.05, utilizando el software estadístico INFOSTAT Versión 2011 [19] posteriormente se determino el coeficiente de correlacion de Pearson y el coeficinte de determinacion.

\section{Resultados y discusión}

\section{Determinacion de Polifenoles totales}

Los resultados de los análisis de contenido de polifenoles totales (PFT) se presentan en el cuadro 1. El contenido de polifenoles totales resultó 14.16 y 9.40 (mg de ácido gálico GAE/g) para el té de hojas de camote y el té verde respectivamente, el análisis estadístico muestra diferencias significativas entre los dos $(F=19.71 \quad p=0.0113)$. De acuerdo a éstos resultados el té de hojas de camote presenta mayor contenido de polifenoles totales frente al té verde comercial.

En un estudio similar se analizaron extractos acuosos a $90^{\circ} \mathrm{C}$ de 34 muestras de café comercial puro y mezclado en Panamá, encontrando valores para café mezclado en un rango de 11.17 a $16.10 \mathrm{mg} \mathrm{GAE/g} \mathrm{[13],} \mathrm{también} \mathrm{se} \mathrm{evaluaron} \mathrm{infusiones} \mathrm{o} \mathrm{tés} \mathrm{de} \mathrm{diferentes} \mathrm{plantas} \mathrm{en} \mathrm{Brasil}$ (té negro, té verde, infusión de mate, menta, manzanilla, anís, hinojo entre otros) resultando que el contenido de fenoles fue desde no determinado hasta 46.6 mg GAE/g de muestra [12], por otro lado se evaluó un extracto acuoso de hojas de maracuyá a temperatura de ebullición obteniéndose $8.3 \mathrm{mg}$ GAE/g [20], nuestros resultados coinciden e incluso están por encima de estos resultados, lo que sería un indicador que el té de hojas de camote morado es una excelente fuente de polifenoles totales.

Cuadro 1. Contenido de polifenoles totales (mg GAE /g)

\begin{tabular}{|c|c|}
\hline MUESTRA & PFT $(\mathrm{mg} \mathrm{GAE} / \mathrm{g})^{\star}$ \\
\hline TÉ DE CAMOTE & $14.16 \pm 1.63^{\mathrm{a}}$ \\
\hline TÉ VERDE & $9.40 \pm 0.67^{\mathrm{b}}$ \\
\hline
\end{tabular}

*Los datos son el promedio de tres repeticiones. Letras iguales corresponden a grupos similares de acuerdo al análisis de medias (Tukey, $\mathrm{p}<0.05$ ). 


\section{Determinacion de Capacidad Antioxidante TEAC ABTS}

Los resultados de la capacidad antioxidante equivalente Trolox TEAC ABTS, se muestran en el cuadro 2. En la evaluacion se obtuvo 8.61 y 7.98 ( $\mu \mathrm{mol}$ Trolox $/ \mathrm{mL})$ para el té de hojas de camote y el té verde respectivamente, El análisis estadístico indica diferencias significativas entre las muestras ( $F=50.70 \quad p=0.0021)$, lo cual demuestra que el té de hojas de camote es significativamente mayor frente al té verde en cuanto a su capacidad antioxidante; en un estudio similar se evaluó la capacidad antioxidante de infusiones comerciales de hierbabuena, limón, manzanilla, árnica, boldo y té verde obteniendo resultados en un rango de 0.55 - 4.90( $\mu \mathrm{mol}$ Trolox/mL) [21], por otro lado también se evaluaron 13 muestras de tés herbales entre ellas boldo, rosa mosqueta y baylahuen presentando de 152 a $1056 \mu \mathrm{mol}$ eq.Tx/150 mL (1.01 a $7.04 \mu \mathrm{mol}$ eq.Tx/mL) [22], de acuerdo a estos resultados el té de hojas de camote morado presentaría una buena actividad antioxidante, indicando su potencial actividad antioxidante.

Cuadro 2. Capacidad antioxidante TEAC ABTS ( $\mu \mathrm{mol}$ Trolox/mL)

\begin{tabular}{|c|c|c|}
\hline MUESTRA & TEAC ABTS $(\mu \mathrm{mol} \text { Trolox } / \mathrm{mL})^{*}$ & \% Inhibición \\
\hline TÉ DE CAMOTE & $8.61 \pm 0.06^{\mathrm{a}}$ & $75.78 \pm 0.27$ \\
\hline TÉ VERDE & $7.98 \pm 0.14^{\mathrm{b}}$ & $72.08 \pm 0.75$ \\
\hline
\end{tabular}

*Los datos son el promedio de tres repeticiones. Letras iguales corresponden a grupos similares de acuerdo al análisis de medias (Tukey, $\mathrm{p}<0.05$ ).

\section{Determinacion de Capacidad Antioxidante TEAC DPPH}

Los resultados de la capacidad antioxidante equivalente Trolox TEAC DPPH, se presentan en el cuadro 3. Obteniendo resultados de 25.16 y 25.63 ( $\mu$ mol Trolox/g) en el té de camote y té verde respectivamente, El análisis estadístico muestra que no existen diferencias entre ambas muestras ( $F=1.86 p=0.2446$ ), lo que indica que la capacidad antioxidante en el té de hojas de camote es muy similar al té verde, con porcentajes de inhibicion de los extractos de 68 y 69 $\%$ frente al radical libre DPPH demostrando su similitud. En un estudio se ha determinado la actividad antioxidante en muestras de extractos acuosos de 34 tipos de café puros y mezclados comerciales de Panamá encontrándose para 16 tipos de café mezclados una actividad antioxidante de 0.025 a 0.060 mmol de equivalente Trolox (TE)/g (25 a 60 umol Trolox/g) con $\mathrm{DPPH}$ [13] encontrándose nuestros resultados dentro de ese rango; así mismo se han analizado extractos acuosos de siete plantas medicinales de la familia de Lamiaceae entre ellas orégano, menta, tomillo, salvia, romero etc., obteniendo una actividad antioxidante de 6.05 a 9.92 umol Trolox/g con DPPH [23], estos resultados nos llevan a inferir que el té de camote morado presentaría considerable efectividad antioxidante similar al del té verde.

Existen muchos estudios que utilizan el método TEAC DPPH para determinar la capacidad antioxidante de compuestos puros y extractos de plantas, pero es difícil compararlos con nuestros resultados porque se aplican diferentes condiciones de estudio y análisis [24].

Cuadro 3. Capacidad antioxidante TEAC DPPH ( $\mu \mathrm{mol}$ Trolox/g)

\begin{tabular}{|c|c|c|}
\hline MUESTRA & TEAC DPPH $(\mu \mathrm{mol} \text { Trolox } / g)^{\star}$ & \% Inhibición \\
\hline TÉ DE CAMOTE & $25.16 \pm 0.13^{\mathrm{a}}$ & $68.63 \pm 0.34$ \\
\hline TÉ VERDE & $25.63 \pm 0.58^{\mathrm{a}}$ & $69.88 \pm 1.55$ \\
\hline
\end{tabular}

*Los datos son el promedio de tres repeticiones. Letras iguales corresponden a grupos similares de acuerdo al análisis de medias (Tukey, $p<0.05$ ). 
Correlación de Pearson entre polifenoles totales y la actividad antioxidante TEAC-ABTS.

Para probar si existe correlación entre polifenoles totales y la capacidad antioxidante TEAC ABTS, se ha determinado la correlación de Pearson, observándose una correlación significativa (figura 3) con un coeficiente de correlación $r=0.9129$ y un coeficiente de determinación $r^{2}$ $=0.8334$, lo que demuestra que a mayor contenido de polifenoles presentes en los productos se tiene mayor capacidad antioxidante.

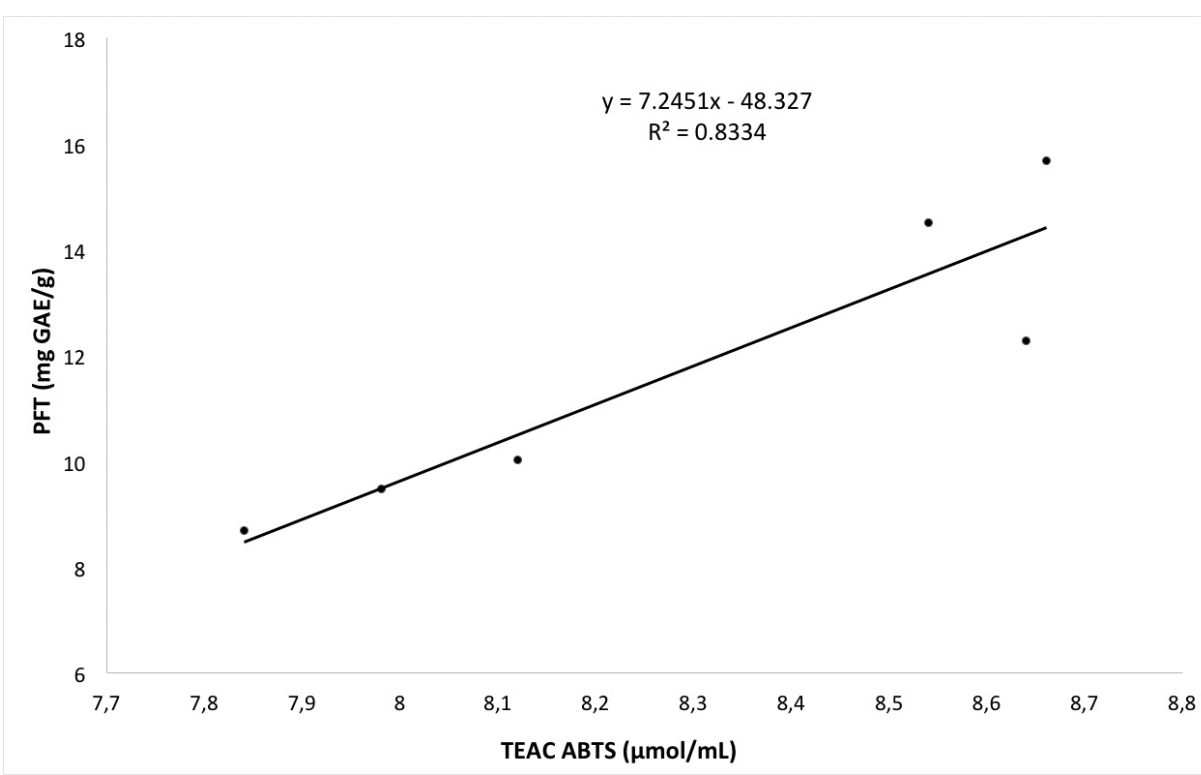

Figura 3. Correlación lineal entre polifenoles totales y actividad antioxidante TEAC-ABTS

\section{Conclusiones}

El té de hojas de camote morado frente al té verde, presentó actividad antioxidante efectiva por el contenido de polifenoles totales, asi como por la prueba analitica del TEAC ABTS, en el caso de la capacidad antioxidante TEAC DPPH ambos presentaron similitudes, demostrando que es un producto con alto poder bioactivo, lo que implica su mayor poder antioxidante, cercano y/o similar al té verde, aunque esto podria potenciarse mejorando las condiciones y tiempos de extracción.

La correlacion de Pearson indica una alta correlacion entre valores de polifenoles y actividad antioxidante por TEAC ABTS.

\section{Agradecimientos}

Los autores agradecen a la Escuela Académico Profesional de Ingenieria Agroindustrial, Facultad de Ingeniería, Universidad Nacional Micaela Bastidas de Apurímac por las facilidades brindadas para la ejecución de este trabajo de investigación, y al Vicerrectorado de Investigacion de la Universidad Nacional Micaela Bastidas de Apurímac por el apoyo en su difusion. 


\section{Referencias}

[1] A. Sani, W.O. Abdullah, T. Juhana, and M.A. Elkadi "Extraction, characterization and total phenolic content of local (Malaysian) green sweet potato (Ipomoea batatas) leaves". International Journal of Science Commerce and Humanities, vol. 2, n 5, pp. 175-182, 2014.

[2] L.V. An, "Sweet potato leaves for growing pigs: Biomass yield, digestion and nutritive value". Doctor's dissertation. Swedish University of Agricultural Sciences, Uppsala, 2004. ISSN 1401-6249, ISBN 91-576-6750-0.

[3] A.W. Mwanri, "The potential of sweet potato (Ipomoea batatas L.) leaves in contribution to vitamins and minerals in the diet of children in Tanga-Tanzania", Thesis Degree of Masters of Science in Applied Human Nutrition of the University of Nairobi, Kenya, 2003.

[4] G. Nkongho, A. Achidi, N. Ntonifor, N. Numfor, B. Dhinga, L. Jackai and C. Bonsi, "Sweet pototoes in Cameroon: Nutritional profile of leaves and their potential new use in local foods", African Journal of Agricultural Research, vol. 9, n 18, pp. 1371-1377, 2014 DOI 10.5897/AJAR2014.8611.

[5] H. Sun, T. Mu, L. Xi, M. Zhang and J. Chen, "Sweet potato (Ipomoea batatas L.) leaves as nutritional and functional foods", Food Chemistry, vol.156, pp. 380-389, 2014. http://dx.doi.org/10.1016/j.foodchem.2014.01.079

[6] M. Islam, M. Yoshimoto, N. Terahara and O. Yamakawa, "Anthocyanin compositions in sweetpotato (Ipomoea batatas L.) leaves”, Biosci. Biotechnol. Biochem., vol.66, n¹1, pp. 2483-2486, 2002.

[7] S.M. Hue, A.N. Boyce and Ch. Somasundram, "Antioxidant activity, phenolic and flavonoid contents in the leaves of different varieties of sweet potato (Ipomoea batatas)" Australian Journal of Crop Science, vol. 6, $\mathrm{n}^{\circ}$ 3, pp. 375-380, 2012.

[8] A. Ghasemzadeh, V. Omidvar and H.Z.E. Jaafar, "Polyphenolic content and their antioxidant activity in leaf extract of sweet potato (Ipomoea batatas)", Journal of Medicinal Plants Research, vol. 6, n 15, pp. 2971-2976, 2012. DOI: 10.5897/JMPR011.1353

[9] V.D. Truong, R.F. Mcfeeters, R.T. Thompson, L.L. Dean and B.Shofran, "Phenolic acid content and composition in leaves and roots of common commercial sweetpotato (Ipomea batatas L.) cultivars in the United States", Journal Of Food Science, vol. 72, nº 6, pp. 343-349, 2007. doi: 10.1111/j.1750-3841.2007.00415.x

[10] J. Ramírez-Godínez, J. Jaimez-Ordaz, J. Añorve-Morga, V. Salazar-Pereda, Castañeda-Ovando, G. GonzálezOlivares y E. Contreras-López, "Determinación de actividad antioxidante en extractos acuosos de cedrón (Aloysia triphylla)" Investigación y Desarrollo en Ciencia y Tecnología de Alimentos, vol. 1, pp. 824-829, 2016.

[11] V. V. Melchor-Sandoval, "Procesamiento tecnológico para la obtención de té verde (Camellia sinensis): Determinación de su actividad antioxidante y cuantificación de flavanoles por HPLC". Tesis de licenciatura Facultad de Industrias Alimentarias Universidad Nacional Agraria de la Selva, Tingo María, Perú, 2002.

[12] R.A. Moraes-de-Souza, T.L.C. Oldoni, M.A.B. Regitano-d'Arce, S.M. Alencar, "Actividad antioxidante y compuestos fenólicos en infusiones herbarias consumidas en Brasil”, Cienc. Tecnol. Aliment. vol. 6, no 1, pp. 41-47, 2008.

[13] A. Vega, J.A. De León y S.M. Reyes, "Determinación del contenido de polifenoles totales, flavonoides y actividad antioxidante de 34 cafés comerciales de Panamá", Información Tecnológica, vol. 28, n 4, 2017. doi: 10.4067/S0718-07642017000400005

[14] V.L. Singleton, R. Orthofer, and R.M. Lamuela-Raventos "Analysis of total phenols and other oxidation substrates and antioxidants by means of Folin-ciocalteu reagent" Methods in enzymology, vol. 299, pp. 152-178, 1999.

[15] R. Re, N. Pellegrini, A. Proteggente, A. Pannala, M. Yang, and C. Rice-Evans, "Antioxidant activity applying an improved ABTS radical action decolorization assay". Free radical biology and medicine, vol. 26, n॰ 9, pp. 1231-1237, 1999.

[16] N. Razali, R. Razab, S.M. Junit, and A.A. Aziz, "Radical scavenging and reducing properties of extracts of cashew shoots (Anacardium occidentale)", Food chemistry, vol. 111, n 1, pp. 38-44, 2008.

[17] D. M. Juli-Mamani y E. Arias-Jordán, "Antocianinas polifenoles totales y capacidad antioxidante en dos clones de papa nativa del género Solanum de pulpa azul y roja". Tesis de licenciatura Facultad de Ingeniería Agroindustrial, Universidad Nacional San Antonio Abad del Cusco, Sicuani-Cusco, Perú, 2011.

[18] M. Barragán-Condori, y Aro Aro, J. M., "Determinación del efecto de procesos de cocción en papas nativas pigmentadas (Solanum tuberosum spp. andigena) sobre sus compuestos bioactivos", Revista de Investigaciones Altoandinas, vol. 19, pp. 47-52, 2017. http://dx.doi.org/10.18271/ria.2017.254

[19] J. A. Di Rienzo, F. Casanoves, M. G. Balzarini, L. Gonzalez, M. Tablada y C. W. Robledo, INFOSTAT, 2011.

[20] J. K. da Silva, C.B. Betim-Cazarin, T. C. Colomeu, Â.G. Batista, L.M.M. Meletti, J.A. Rizzato-Paschoal, S. Bogusz-Júnior, M. Fontes-Furlan, F.G. Reyes-Reyes, F. Augusto, M. R. Maróstica-Júnior, and R. de Lima- 
Zollner "Antioxidant activity of aqueous extract of passion fruit (Passiflora edulis) leaves: In vitro and in vivo study" Food Research International, vol. 53, pp. 882-890, 2013.http://dx.doi.org/10.1016/j.foodres.2012.12.043

[21] E. E. Muñoz-Velázquez, K. Rivas-Díaz, M. G. F. Loarca-Piña, S. Mendoza-Díaz, R. Reynoso-Camacho y M. Ramos-Gómez, "Comparación del contenido fenólico, capacidad antioxidante y actividad antiinflamatoria de infusiones herbales comerciales" Revista Mexicana de Ciencias Agrícolas, vol. 3, n³ 3, pp. 481-495, 2012.

[22] H. Speisky, C. Rocco, C. Carrasco, E. A. Lissi and C. López-Alarcón, "Antioxidant Screening of medicinal herbal teas" Phytotheraphy research,https://www.ncbi.nlm.nih.gov/pubmed/16619353 vol. 20, n 6, pp. 462-467, 2006. DOI:10.1002/ptr.1878

[23] N. Gougoulias and N. Mashev, "Antioxidant activity and polyphenols content of some herbal teas of Lamiaceae family from Greece and Bulgaria" Oxidation Communications vol. 38, n¹, pp. 25-34, 2015.

[24] V.G. Hartwig, L.A. Brumovsky, R.M. Fretes, L. and Sánchez-Boado, "A novel procedure to measure the antioxidant capacity of yerba maté extracts", Ciênc. Tecnol. Aliment., Campinas, vol. 32, n 1, pp. 126-133, 2012. DOI: http://dx.doi.org/10.1590/S0101-20612012005000022. 